\title{
PVDF Hollow Fiber and Nano Fiber Membranes for Fresh Water Reclamation using Membrane Distillation
}

*Lijo Francis, Noreddine Ghaffour, Ahmad Alsaadi, Suzana P. Nunes, Gary L. Amy

Water Desalination and Reuse Center, King Abdullah University of Science and Technology (KAUST), 23955-6900 Thuwal, Kingdom of Saudi Arabia

*Corresponding author email: lijo.francis@ kaust.edu.sa; Phone: +966-128082196

\begin{abstract}
Polyvinylidine fluoride (PVDF) hollow fiber and nanofibrous membranes are engineered and successfully fabricated using dry-jet wet spinning and electrospinning techniques, respectively. Fabricated membranes are characterized for their morphology, average pore size, pore size distribution, nanofiber diameter distribution, thickness and water contact angle. Direct contact membrane distillation (DCMD) performances of the fabricated membranes have been investigated by using a locally designed and fabricated, fully automated MD bench scale unit and DCMD module. Electrospun nanofibrous membranes showed a water flux as high as $36 \mathrm{~L} \mathrm{~m}^{-2} \mathrm{~h}^{-1}$ whereas hollow fiber membranes showed a water flux of $31.6 \mathrm{~L} \mathrm{~m}^{-2} \mathrm{~h}^{-1}$, at a feed inlet temperature of $80^{\circ} \mathrm{C}$ and at a permeate inlet temperature of $20^{\circ} \mathrm{C}$.
\end{abstract}

Keywords: Eelectrospinning, Nanostructured membranes; Hollow fiber membranes; Water vapour flux; Membrane distillation 


\section{Introduction}

MD is a thermally driven membrane-based separation process, considered as one of the technologies that are emerging as an alternative desalination processes. MD utilizes a hydrophobic, macro porous membrane as a contactor to achieve separation by liquid-vapor equilibrium [1]. The pre-heated feed solution is brought into contact with the membrane which allows only the water vapor to go through the membrane pores so that it condenses on the other side of the membrane. This vapor is driven across the membrane by the difference in the partial vapor pressure maintained at the two sides of the membrane. Conventional desalination technologies such as Multi Stage Flash distillation (MSF) and Reverse Osmosis (RO) are not only highly energy intensive processes but they require huge investment cost and large footprint; whereas MD operates at ambient pressure and lower temperatures $\left(30-70^{\circ} \mathrm{C}\right)$ so that any low grade heat source (solar, waste heat and geo thermal) can be sufficient for its operation. Moreover the scalability, inexpensive polymeric materials for the installation, and theoretically $100 \%$ salt rejection, attracts the MD as an attractive desalination process.

The major configurations that have been employed in MD process are direct contact membrane distillation (DCMD), air gap membrane distillation (AGMD), vacuum membrane distillation (VMD) and sweeping gas membrane distillation (SGMD). In all configurations, the hot feed solution will be in direct contact with the membrane. In DCMD, both hot and cold streams are in direct contact with the membrane. In AGMD, a stagnant air gap is maintained between the membrane and a condensation surface. In VMD and SGMD, vacuum and a cold inert gas are passed through the permeate side, respectively, so that the vapor coming across the membrane from the 
feed is condensed outside the membrane module [2]. Apart from these conventional MD configurations, some other configurations such as permeate gap membrane distillation (PGMD) and material gap membrane distillation (MGMD) are also reported recently $[3,4]$.

Electrospinning is a versatile technique for the fabrication of macro porous nanofiber membranes and composite membranes for variety of applications such as biomedical applications, energy harvesting, and filtration applications [5-9]. Nanofiber membranes are those made of fibers with diameters less than 1,000 nm. A wide range of polymeric materials have been explored for the fabrication of nanostructured membranes and composite membranes [10-13] via electrospinning. Recently researchers have explored the electrospun nanofibrous membranes for the water desalination using MD process [14-20]. Conventional techniques for the fabrication of nanofibers are drawing, phase separation, template synthesis and self-assembly. Researchers have shown great interest in the electrospinning technique for the fabrication of nanofibers because of the unique advantages of electrospinning over other techniques. Electrospinning method is convenient to process, reproducible, scalable and the dimensions of the fibers can be engineered by controlling the process parameters during the process [10].

Hollow fiber membranes are also called tubular membranes (in my opinion membranes called tubular are the inorganic ones, e.g. ceramic, zircon... they are rigid. Please check again and revise) with outer diameter less than $3 \mathrm{~mm}$ and with a thickness of less than $300 \mu \mathrm{m}$, and they have been used in wide range of applications especially in the liquid/gas phase separation [any reference?]. They have high surface area to volume ratio and are self-supported and easy to fabricate. MD researchers have been studied different hollow fiber and flat sheet membrane materials such as 
PVDF, poly propylene (PP), poly ethylene (PE) and poly tetra fluoro ethylene (PTFE) [21-25]. Dual layer hydrophobic-hydrophilic membranes and multi bore hollow fiber membranes for MD process have also been reported [26-28]. Recently, fluorinated poly azoles have been synthesized and hollow fiber membranes of those poly azoles have been fabricated and tested for DCMD process [29]. In the present study, PVDF is used for the fabrication of hollow fibers using a specialized?? fabrication machine and nanofiber membranes using electrospinning machine, since PVDF is commercially available, cheap hydrophobic material and easy to process than that of PP, PE or PTFE.

In the present work, commercially available poly vinylidine fluoride (PVDF) is employed to fabricate hydrophobic nanofibrous membranes and hollow fiber membranes. Hollow fibers are fabricated using dry-jet wet spinning, whereas nanofiber membranes are fabricated using electrospinning technique. Fabricated membranes are characterized for their morphology, pore size, thickness and water contact angle. DCMD performances of the fabricated membranes have been tested by using a locally designed and fabricated, fully automated MD bench scale unit and DCMD modules.

\section{Experimental}

PVDF HSV 900 and HSV 761 were kindly provided by Arkema Inc. Ethylene glycol (EG), N-methyl pyrrolidone (NMP) and dimethyl formamide (DMF) were purchased from Sigma Aldrich. PVDF hollow fibers and nanofibers are fabricated by dry-jet wet spinning and electrospinning, respectively. Fig. 1(a) and (b) show the dryjet wet spinning machine and the schematic diagram of the fabrication of hollow fibers, respectively. PVDF hollow fibers were manufactured using a hollow fiber machine (Samwon Engineering Co; LTD, Korea). Dope solution was prepared by 
slowly adding well dried PVDF HSV 900 into NMP/EG in a ratio of 10:1 and stirred continuously with a high speed mechanical stirrer at $70^{\circ} \mathrm{C}$ for $24 \mathrm{~h}$ to get $12(\mathrm{w} / \mathrm{w}) \%$ homogeneous solution of PVDF. EG is a non-solvent which helps in a faster phase separation during spinning and it also act as a pore forming agent. The dope solution was degassed before spinning, and filtered by a metal filter (15 microns) to remove the particles in the solution. The dope solution and deionized water (bore fluid) were fed into the annulus and inner tube of the spinneret separately by using a dope metering pump (Kawasaki khp-1h-0.66/rev) and a lumen metering pump (Laballicance, Flash 100), respectively.
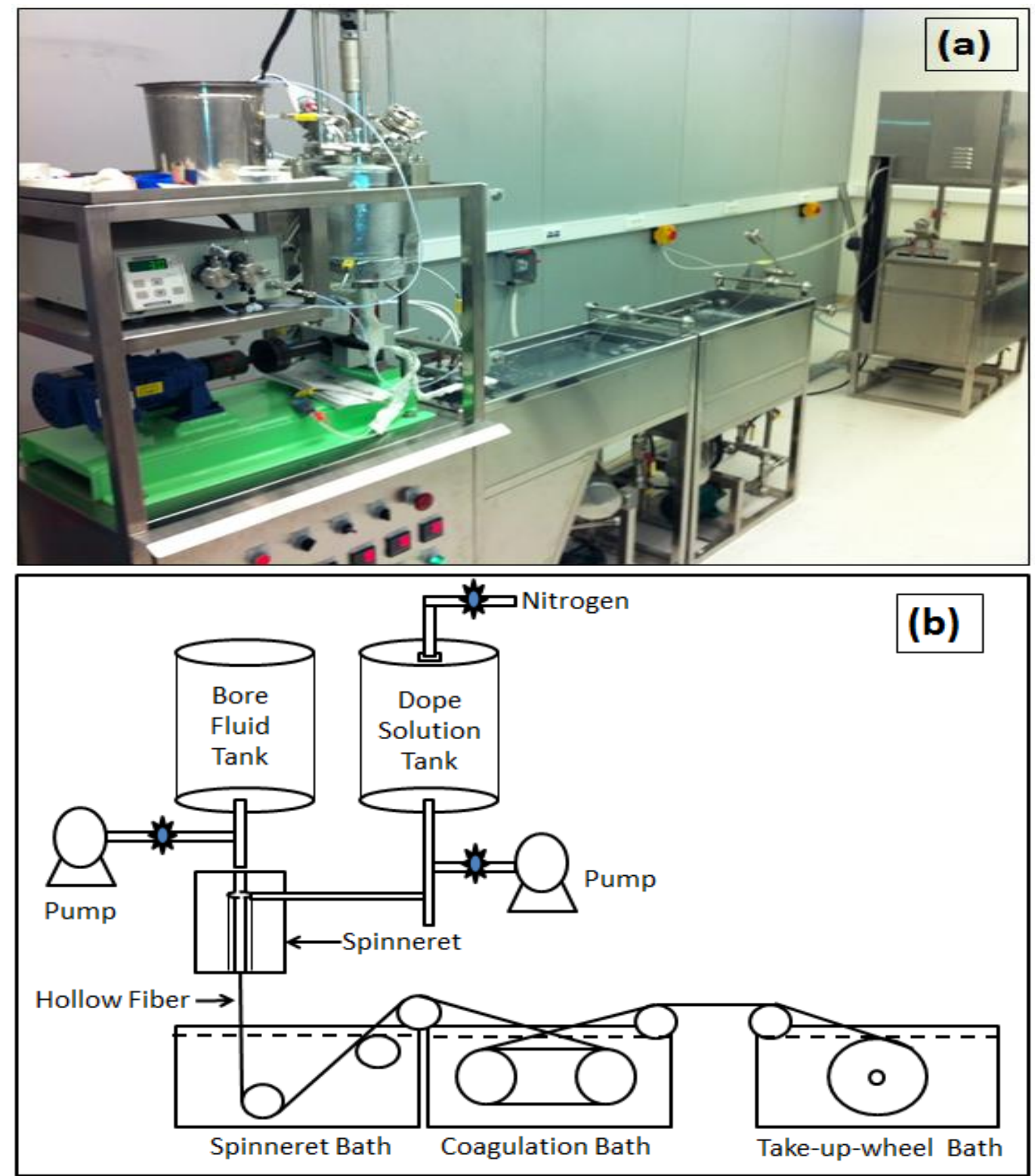
Figure 1. (a) Hollow fiber spinning set-up and (b) Schematic diagram of fabrication of hollow fiber membranes.

Fibers from the outlet of the spinneret were passed through a spinneret bath (water), coagulant bath (water) and collected on a take up wheel. The air gap between the outlet of the spinneret and the spinneret bath was maintained at $5 \mathrm{~cm}$. The as-spun fibers were immersed in water for 3-5 days to remove the residual solvent and stored after freeze drying.

Table 1 shows the fabrication conditions of PVDF hollow fibers.

Table1. Fabrication conditions of PVDF hollow fibers.

\begin{tabular}{|l|l|}
\hline Dope solution & $12(\mathrm{w} / \mathrm{w}) \%$ PVDF HSV 900 in NMP: EG (10:1) \\
Air gap & Tap water \\
$5 \mathrm{~cm}$ \\
External Coagulant & Tap water \\
Bore fluid flow rate & $10 \mathrm{ml} /$ minute \\
Gear pump rotation & $16 \mathrm{rpm}$ \\
\hline
\end{tabular}

Fig. 2 (a) and (b) show the electrospinning machine and schematic diagram of electrospinning for the fabrication of nanofibers membranes, respectively. PVDF HSV 761 was used for the fabrication of nanofibrous membranes. 18 (w/w) \% PVDF in NMP was prepared and used as the dope solution. Dope solution was taken in a syringe and mounted on a syringe pump and the flow rate was set at $1 \mathrm{ml} / \mathrm{hour}$. Aluminium foil mounted on a rotating drum was used a collector to collect nanofibers ejected from the syringe tip. Syringe tip was connected to the positive terminal and rotating drum was connected to the negative terminal of a high voltage power supply (HVPS). HVPS can provide a direct current (DC) voltage from 0 to $50 \mathrm{kV}$. Rotating 
drum was rotated continuously throughout the fabrication with the help of a motor and the drum can also move back and forth with the help of a motor and sensors.
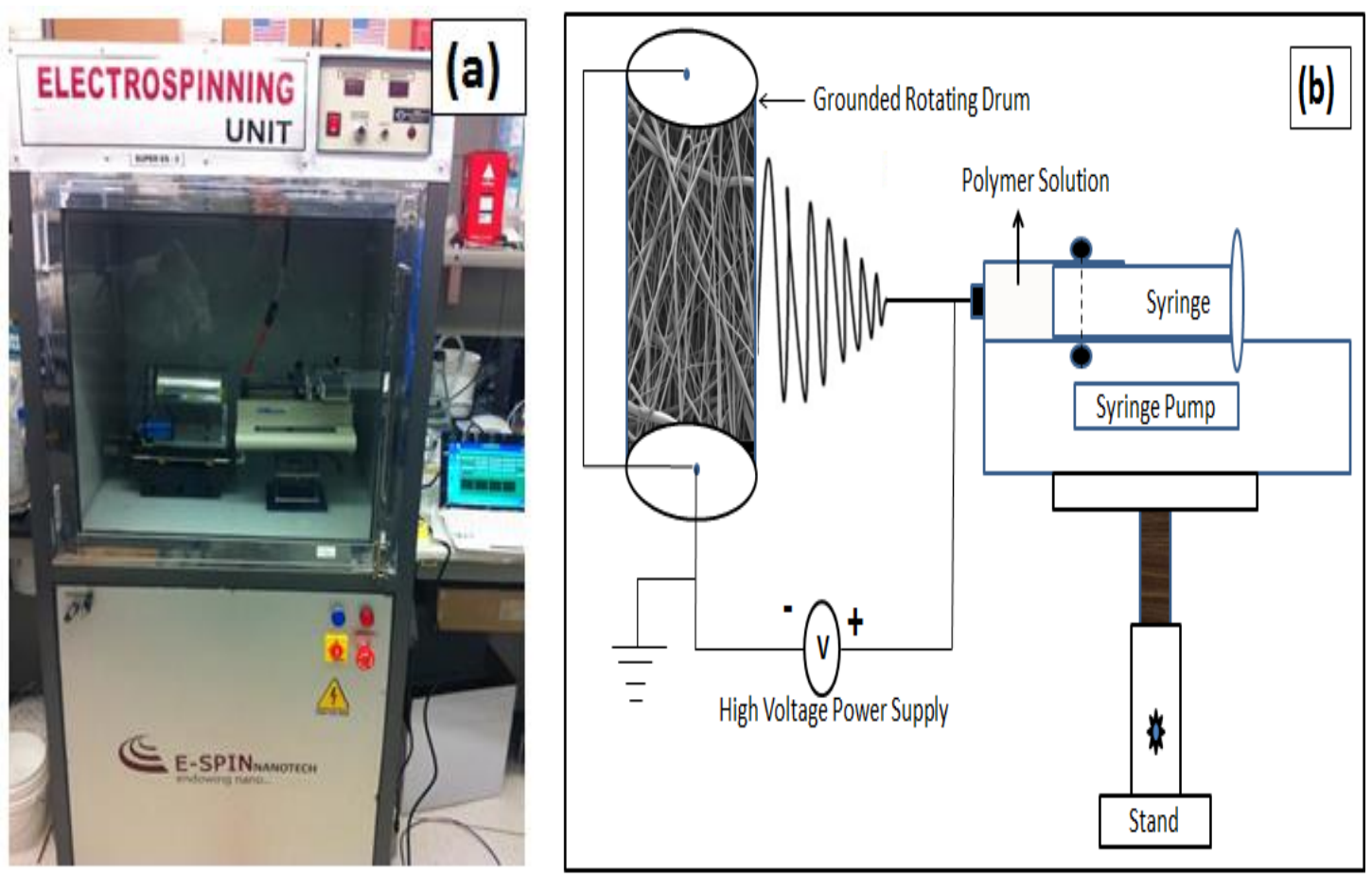

Figure 2. (a) Electrospinning unit and (b) Schematic diagram of fabrication of nanofiber membranes via electrospinning.

While increasing the voltage, after a particular potential (threshold potential), a fast jet of polymeric solution was ejected from the tip of the syringe in the form of nanofibers and collected on the rotating drum. Rotation of the collector drum and it's back and forth movement help to fabricate a uniform membrane. Table 2 shows the fabrication conditions of electrospun PVDF nanofibers.

Table 2. Fabrication conditions of electrospun PVDF nanofibers.

\begin{tabular}{|l|l|}
\hline Dope solution & $18(\mathrm{w} / \mathrm{w}) \%$ PVDF HSV 761 in NMP \\
Dope flow rate & $16.66 \mu \mathrm{L} / \mathrm{hour}$ \\
Applied potential & $25 \mathrm{kV}$ \\
Distance between electrodes & $15 \mathrm{~cm}$ \\
Relative humidity & $65-70 \%$ \\
\hline
\end{tabular}


A thin gold layer was deposited on membrane samples using a Sputter Coater (Emitech K575 X UK) and surface morphology was observed under field emission scanning electron microscope (FESEM; Quanta 200 FEG system: FEI Co; USA). A IB-FT Gm bH Porolux 1,000 Porometer (Germany) was used to measure the nominal pore size and pore size distribution of membrane samples by wet-up/dry-up method with the help of an Automated Capillary Flow Porometer system software. A Attension KSV instruments T 301 (Finland) was used for measuring the water contact angle of membrane samples. Thicknesses of hollow fiber and nanofibers membranes were measured from the FESEM cross section images using image $\mathrm{J}$ software (USA). Conductivity of permeate and feed solutions were determined by using conductivity meter (Oakton Eutech Instruments, Malaysia). A KSV Sigma 701 Tensiometer (KSV Instruments Ltd., Finland) was employed to measure the dynamic contact angle of hollow fibers whereas static water contact angle of flat sheet nanofiber membranes was measured using KSV 301 Attension (KSV instruments Ltd., Finland). An average of five readings was taken as water contact angle. Schematic of water contact angle measurements of flat sheet nanofiber membranes and hollow fiber membranes are shown in Fig. 3 a and b, respectively.
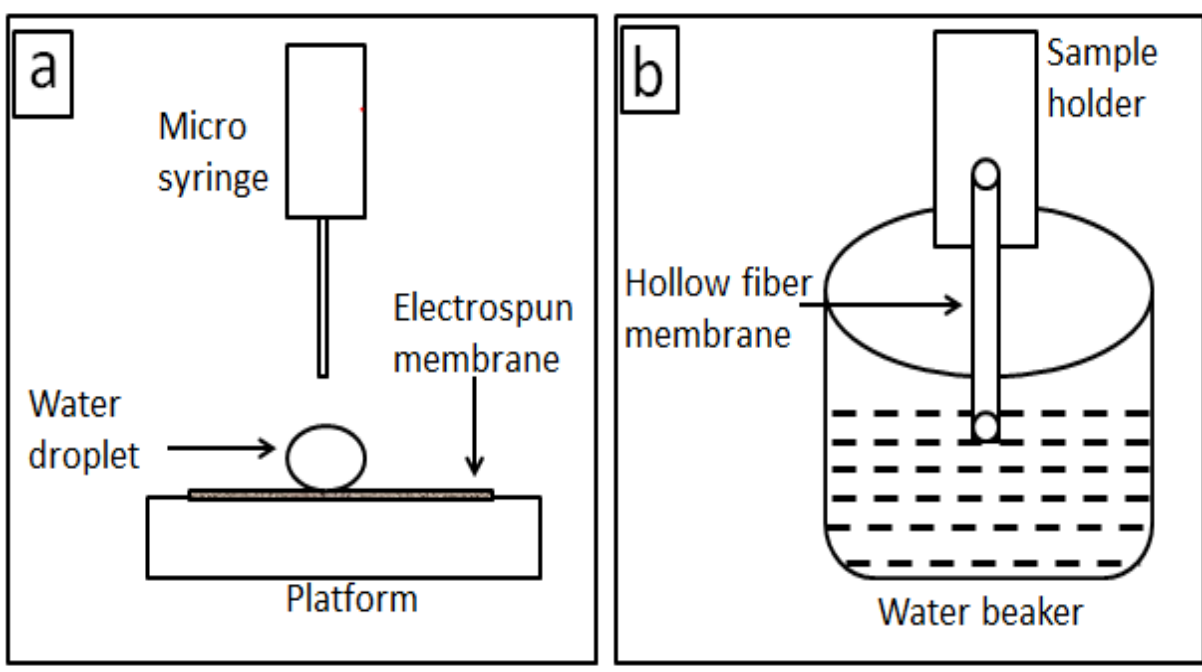
Figure 3. Schematic of water contact angle measurements of (a) electrospun nanofiber membranes, and (b) hollow fiber membranes.

Fig. 4 shows a schematic representation of MD bench scale set up. Membrane module was prepared and mounted on the bench scale set up. Red Seawater was used as feed solution and circulated through one side of the membrane whereas, tap water was circulated along with the permeate from the feed solution, throught the other side of the membrane. Temperature of the permeate side was kept constant by using an electric chiller. Permeate flux was measured at wide range of feed solution temperatures $\left(40-80{ }^{\circ} \mathrm{C}\right)$ and feed temperature was controlled by means of an electric heater. Thermocouples, flow meters and pressure sensors were employed for measuring temperatures, flow rates and pressures, respectively at the entrance and exit points of the membrane module.

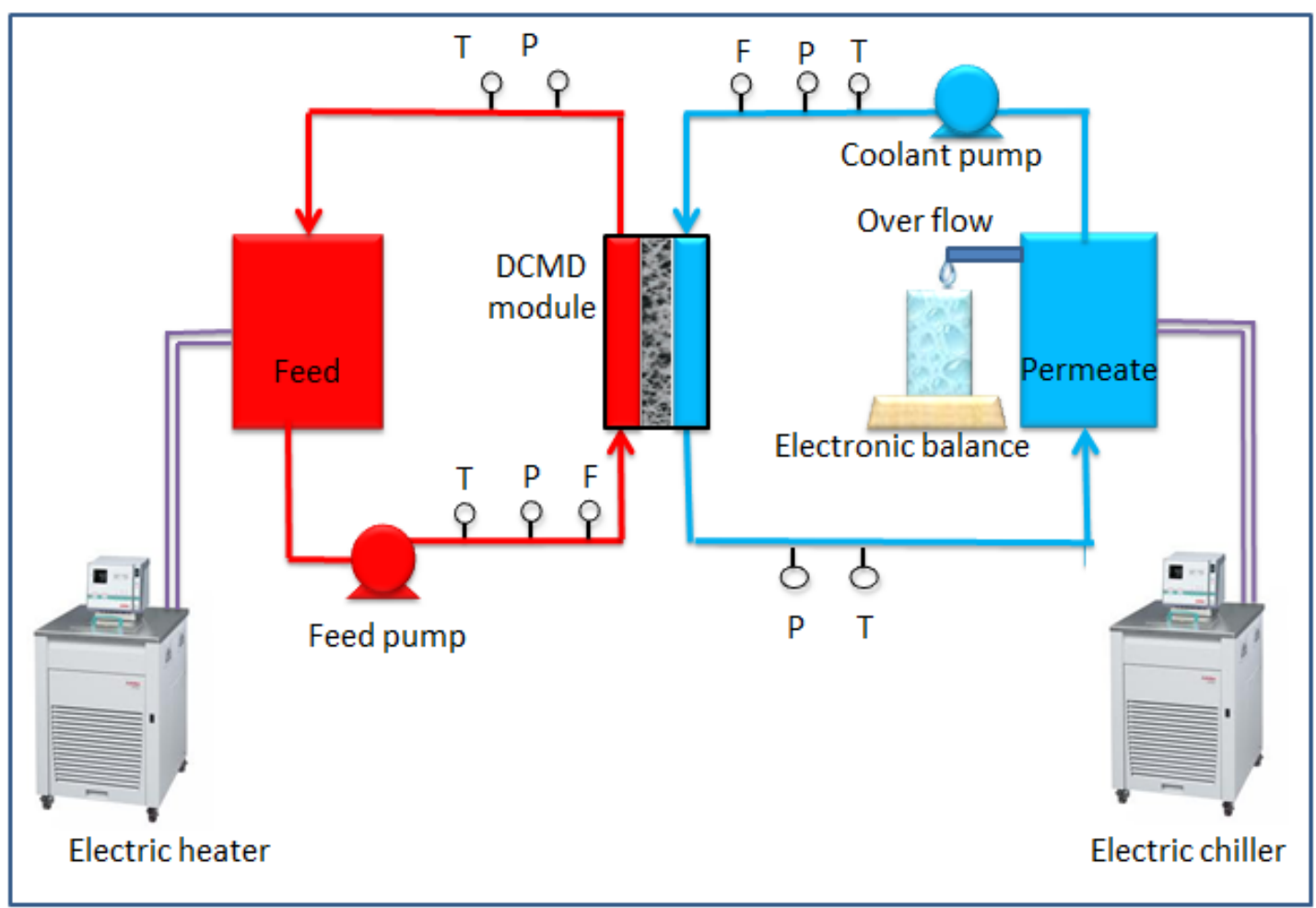

Figure 4. Schematic representation of the bench scale DCMD unit. 
Fig. 5 shows the flat sheet and hollow fiber membrane modules and designs used for DCMD process.

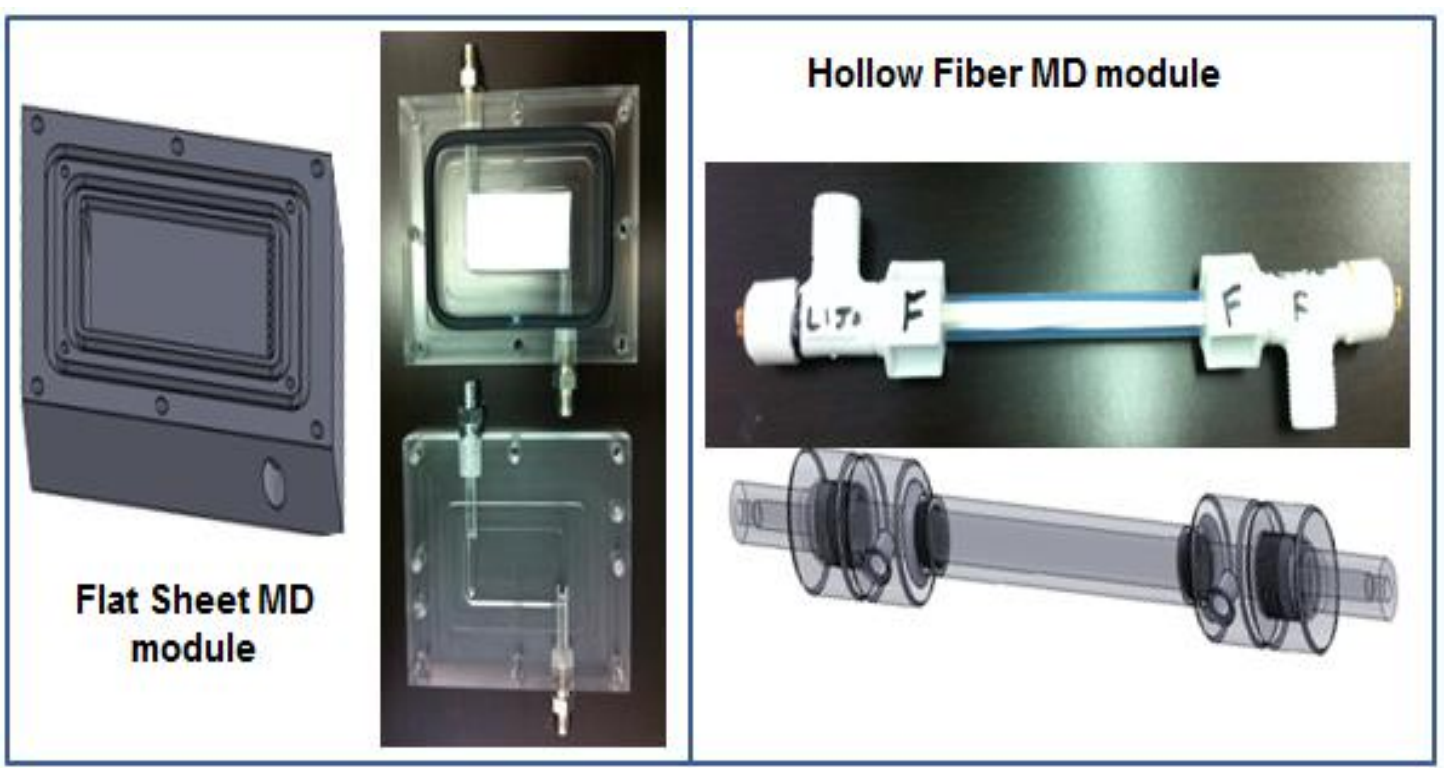

Figure 5. Flat sheet and hollow fiber membrane modules and designs used for DCMD process.

\section{Results and Discussion}

Fig. 6 a shows the FESEM image of cross section of PVDF hollow fiber membrane, Figure 6 (b and c) show the magnified portions of cross section of the hollow fiber, Figure 6 ( $d$ and e) show the surface morphology of PVDF nanofiber membrane, at different magnifications and Figure 6 (f) shows the cross section of the nanofibrous PVDF membrane. FESEM images reveal that the asymmetric hollow fiber membranes have finger like macro voids and a sponge like porous structure in the bulk (Fig. 6 b and c). On the other hand, electrospun nanofibrous membranes are more open structure with high hydrophobicity results in a better mass transfer across the membrane during DCMD process compared to hollow fiber membranes. Water contact angle measurements of electrospun membranes were measured to be $140 \pm 5^{\circ}$ whereas, that of hollow fiber membranes was measured to be $92 \pm 3^{\circ}$. Nonwoven nanofibrous membrane surface morphology with high roughness causes the high water contact angle than hollow fiber membranes with smooth roughness. Presence of 
EG in the hollow fiber membrane dope solution yields smooth surface to the hollow fiber membranes and reduced water contact angle compared to nanofibrous membrane. Thicknesses of hollow fiber and nanofiber membranes were measured to be $115 \mu \mathrm{m}$ and $140 \mu \mathrm{m}$, respectively. Thickness of the membrane has very important role in the heat and mass transfer during MD process. Thicker membranes show high heat and mass transfer resistance.

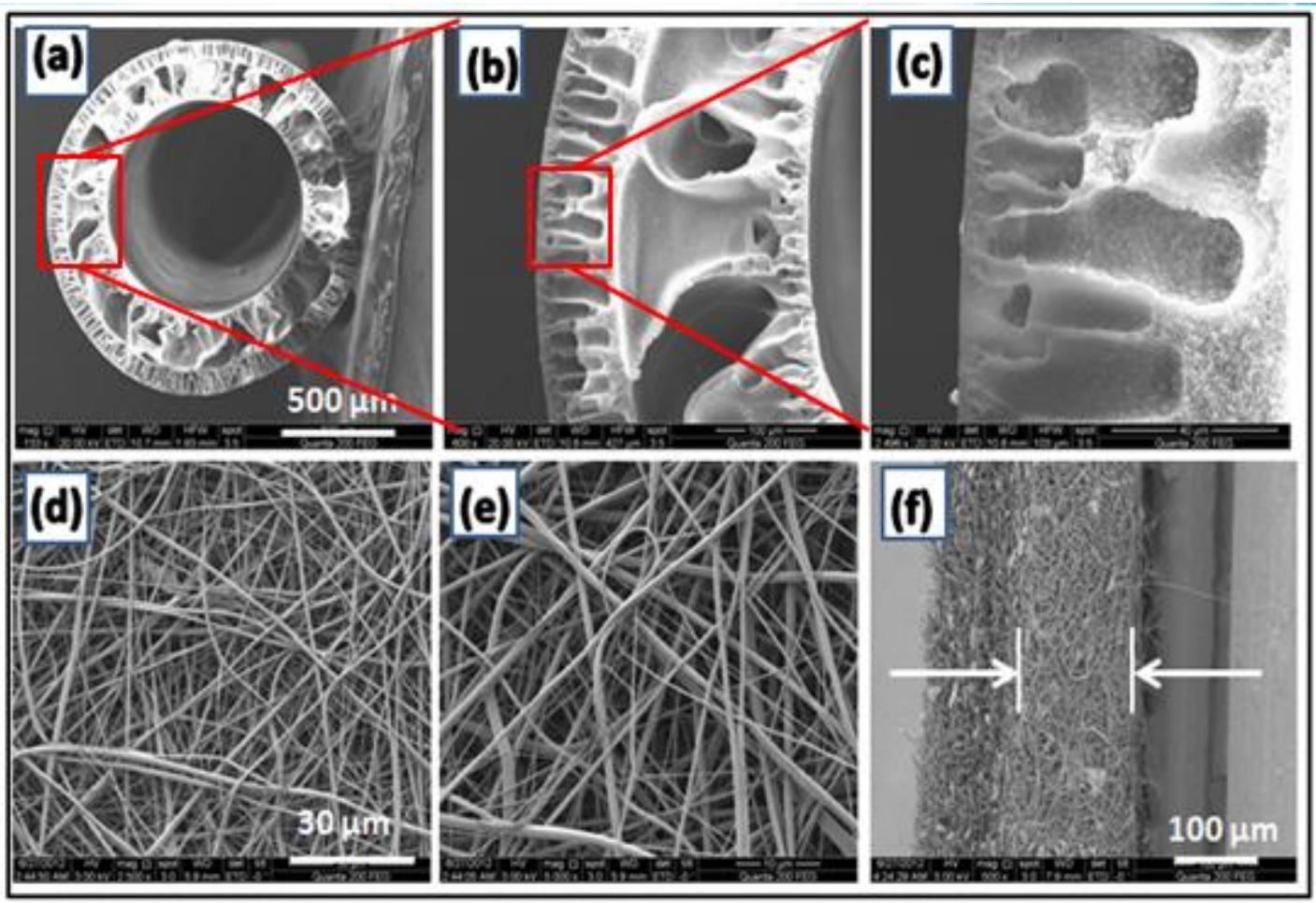

Figure 6. FESEM images of cross section of hollow fiber membrane ( $a, b$ \& c), surface morphology of nano fiber membrane ( $\&$ e e), at different magnifications and cross section of electrospun nano fiber membrane (f).

Fig. 7 shows the fiber diameter distribution of nanofibers and it shows that more than $75 \%$ of the fibers have an average diameter between $200-600 \mathrm{~nm}$. Fig. 8 (a) and (b) show the pore size distribution of hollow fiber and nanofiber membranes, respectively. Hollow fiber membranes have a nominal pore size of $0.1 \mu \mathrm{m}$ and open structured nonwoven nanofiber membranes showed a larger nominal pore size of 1.4 $\mu \mathrm{m}$ and the pore size distribution of electrospun membrane were observed to be narrower than hollow fiber membrane. 


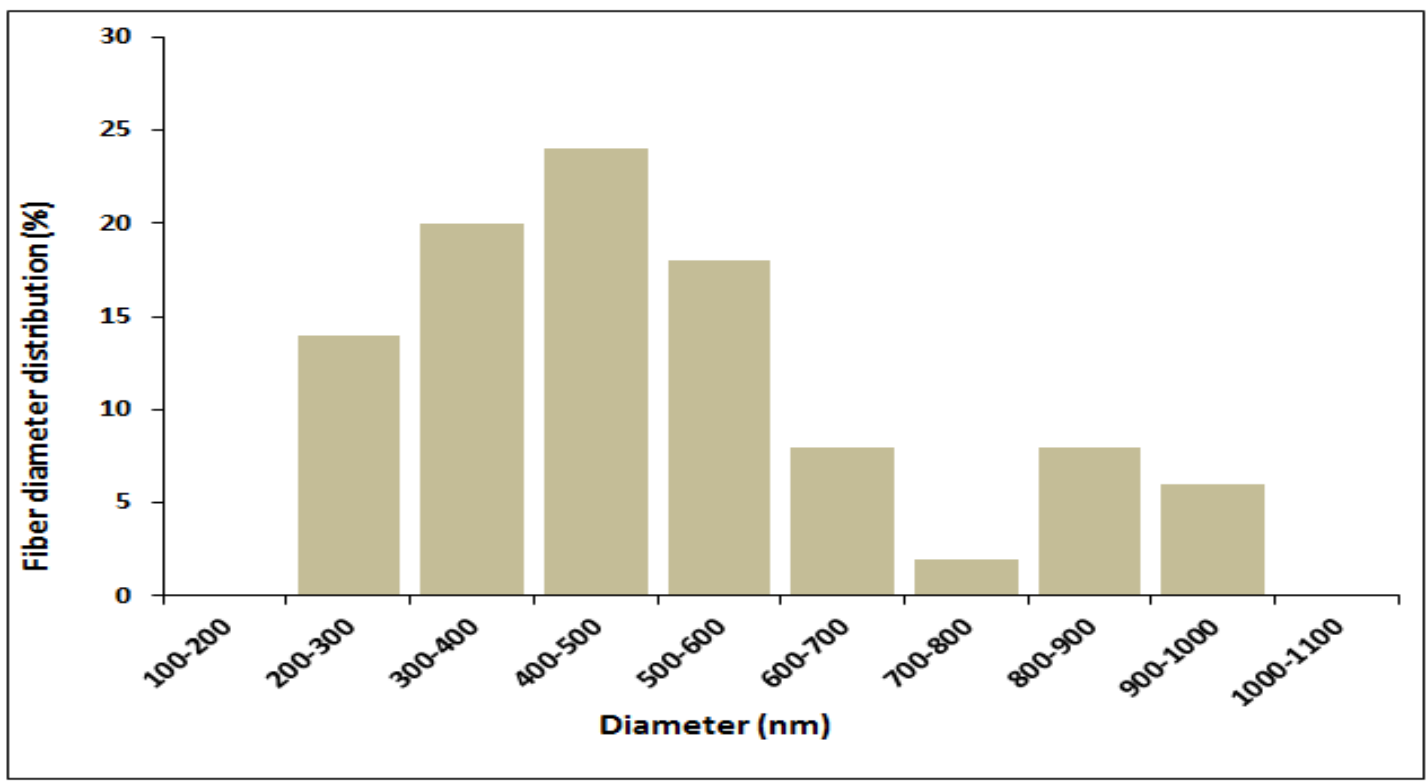

Figure 7. Fiber diameter distribution of electrospun PVDF nanofibers

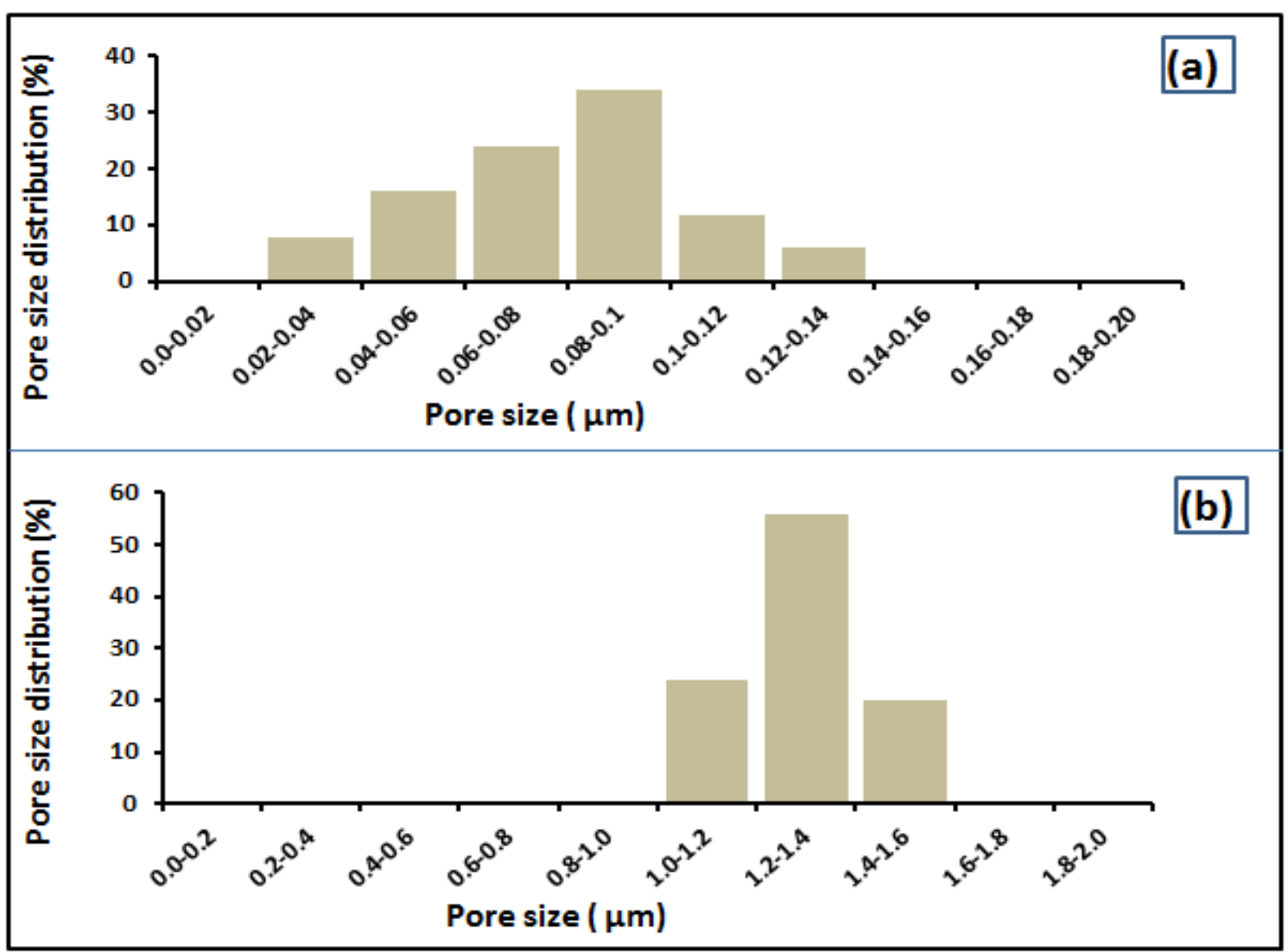

Figure 8. Pore size distribution of (a) hollow fiber membrane and (b) electrospun nanofiber membrane. 
Fig. 9 shows the influence of feed solution temperature on water vapour flux profile at constant permeate flux during DCMD process using hollow fiber and nanofiber membranes. Increase in the feed solution temperature at constant permeate temperature results in the increase in the trans membrane vapour pressure difference and increases the water flux accordingly. Electrospun nanofibrous membranes showed a water flux of $36 \mathrm{~L} \mathrm{~m}^{-2} \mathrm{~h}^{-1}$ whereas hollow fiber membranes showed a water flux of $31.6 \mathrm{~L} \mathrm{~m}^{-2} \mathrm{~h}^{-1}$, at a feed inlet temperature of $80{ }^{\circ} \mathrm{C}$ and at a permeate inlet temperature of $20{ }^{\circ} \mathrm{C}$. These results are comparable with reported studies in the literature using synthetic seawater or distilled water as feed solution. In the present study, the given results are obtained using real Red Seawater as feed solution.

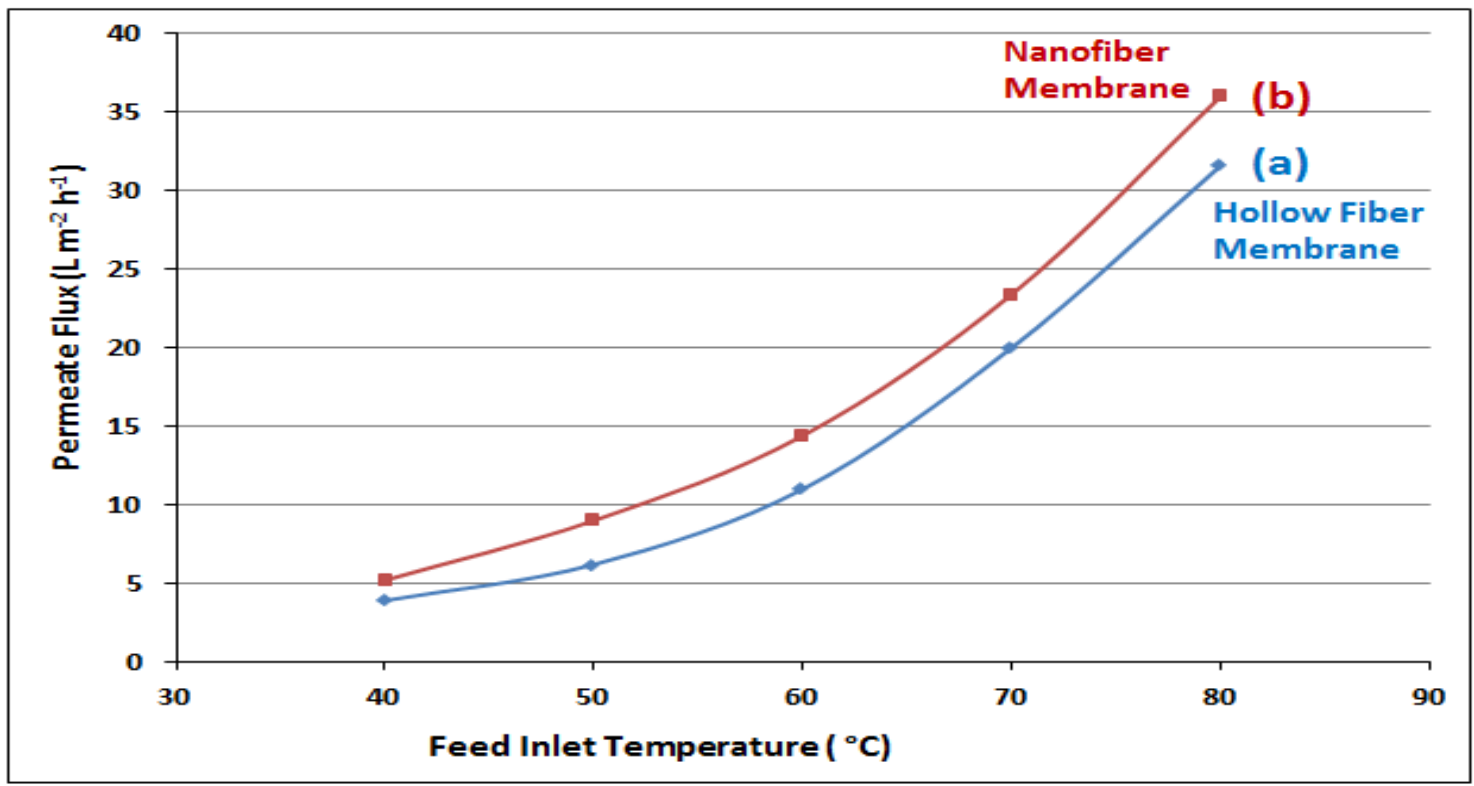

Figure 9. Water vapour flux profile at different feed inlet temperature during DCMD process using (a) hollow fiber membrane and (b) electrospun nanofiber membrane.

Conductivity of the tap water used in the permeate side was measured to be $6 \mu \mathrm{S} / \mathrm{cm}$ and after DCMD process, it was measured to be less than $10 \mu \mathrm{S} / \mathrm{cm}$ in all experiments. Even though, the fabricated PVDF nanofibrous membrane is thicker than hollow fiber membrane, the large pore size and more open and inter connected pore structure of nanofibrous membranes result in $14 \%$ higher water flux compared to 
hollow fiber membrane at a feed inlet temperature of $80{ }^{\circ} \mathrm{C}$. Increased hydrophobicity of electrospun membranes prevent them from pore wetting, regardless of their larger pore size, compared to hollow fiber membranes.

Fig. 10 shows the vapour flux profile at different permeate inlet temperatures during DCMD process using hollow fiber and electrospun nanofiber membranes.

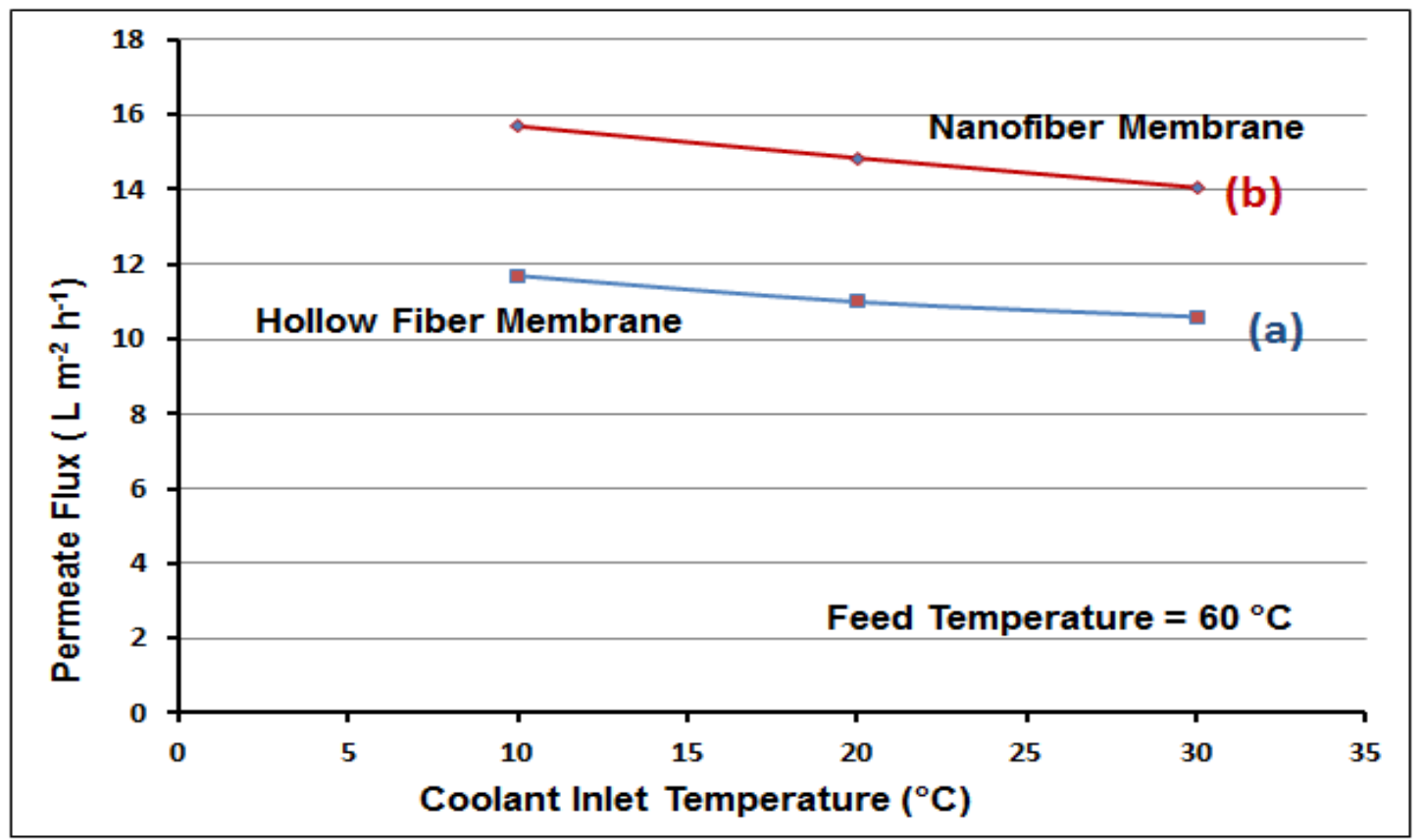

Figure 10. Water vapour flux profile at different coolant inlet temperatures during DCMD process using (a) hollow fiber membrane and (b) electrospun nanofiber membrane.

Trans-membrane vapour pressure difference at lower temperatures is lower than that at higher temperatures and an increase in the water vapour flux by decreasing the coolant temperature at constant feed inlet temperature was observed to be significantly lesser when compared to the increase in the permeate flux while increasing the same degree of feed inlet temperature at constant coolant inlet temperatures.

Most of the reported studies in the area of the MD process investigation use distilled water or Sodium Chloride solution (synthetic seawater) as feed solution. This will not give us a realistic operational condition of the challenges faced in the MD process, 
mainly fouling and scaling which occur in real seawater desalination. Fig. 11 shows the SEM surface morphology of electrospun nanofibers membrane after DCMD process. Since real Red Sea water was used as the feed solution during DCMD process, the possibilities of precipitation of sparingly soluble salts at high temperatures are unavoidable. This will block the membrane pores and eventually flux decline occurs. A detailed investigation to mitigate fouling and scaling during MD process and process optimization is inevitable to enhance the process performance. Several researchers, e.g., Khayet et al. [add reference number]; Bonyadi et al. [add reference number], and Yang et al. [add reference number] reported modified MD membranes with durability and performance improvement of the MD process. Precisely engineered membranes are yet to be developed and essential in terms of large scale fabrication with appropriate characteristics suitable for long term operation.
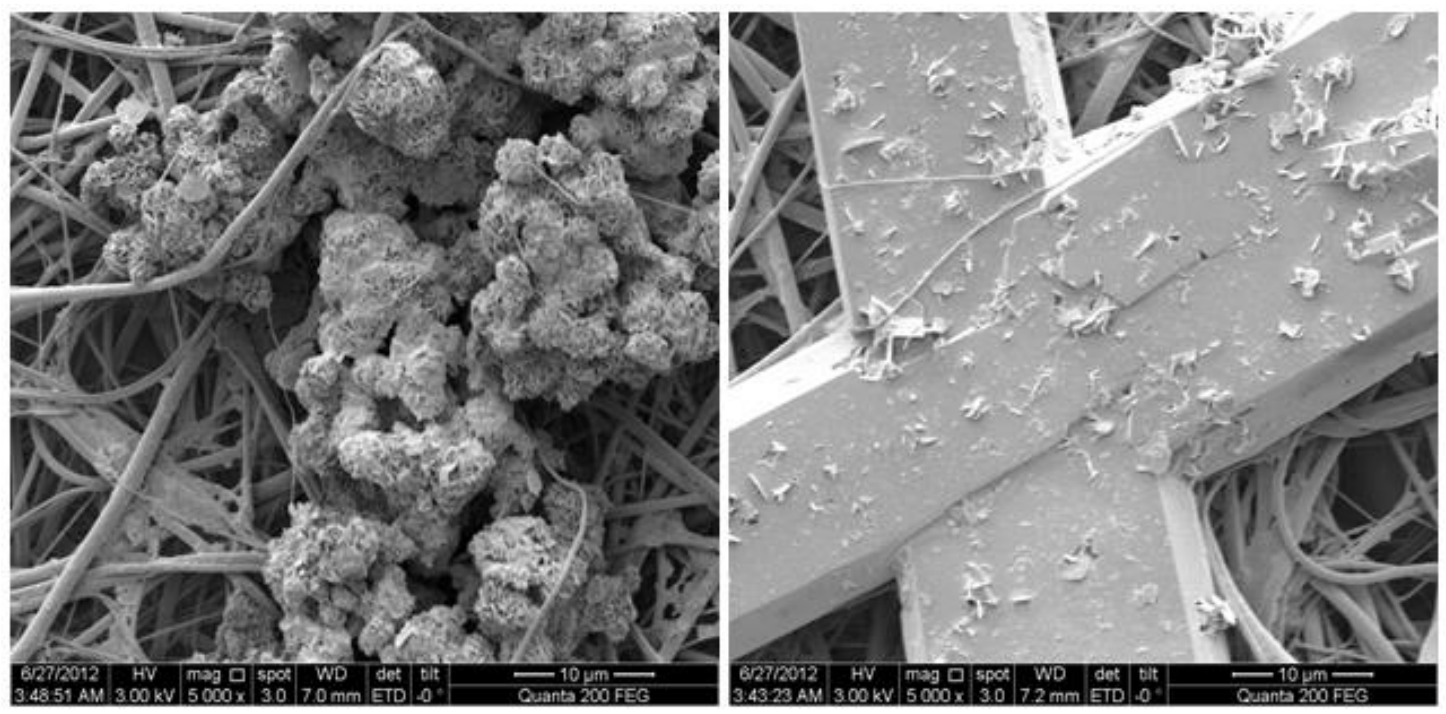

Figure 11. SEM images of electrospun nanofibers membranes after DCMD process showing deposits on the membrane surface.

\section{Conclusion}


PVDF hollow fiber membranes and nanofibers membranes have been successfully fabricated using wet-jet spinning and electrospinning, respectively. Fabricated membranes are characterized and tested for DCMD process and a high salt rejection rate was observed in all experiments. Electrospinning is a good tool for the fabrication of nanostructured membranes, whereas, hollow fiber membranes are selfsupported and easy to fabricate membranes and membrane modules. Nonwoven fibrous morphology of electrospun membranes create surface roughness and increased hydrophobicity, compared to hollow fiber membranes. Electrospun nanofibrous membranes showed a water flux as high as $36 \mathrm{~L} \mathrm{~m}^{-2} \mathrm{~h}^{-1}$ whereas hollow fiber membranes showed a water flux of $31.6 \mathrm{~L} \mathrm{~m}^{-2} \mathrm{~h}^{-1}$, at a feed inlet temperature of $80{ }^{\circ} \mathrm{C}$ and at a permeate inlet temperature of $20{ }^{\circ} \mathrm{C}$. Open structure and interconnected pores in electrospun membranes leads to a $14 \%$ increase in the trans membrane water vapour flux during DCMD process at a $\Delta \mathrm{T}$ of $60^{\circ} \mathrm{C}$. Unavoidable fouling and scaling issues in MD process should be investigated in detail to enhance the process performance.

\section{Acknowledgements}

Authors would like to thank Dr. Francois BEAUME from ARKEMA/Kynar fluoro polymers development for their kind supply of PVDF. Authors would like to thank Meshaal Abdulkareem, Yang Liu and Seraj from electronic workshop at KAUST for their kind assistance in the membrane fabrication setup.

\section{References}


[1] Alsaadi AS, Ghaffour N, Li JD, Gray S, Francis L, Maab H and Amy GL (2013) Modeling of air-gap membrane distillation process: A theoretical and experimental study, Journ Membr Sci 445: 53-65.

[2] Lawson KW and Lloyd DR (1997) Membrane distillation, Journ Membr Sci 124: $1-25$.

[3] Cipollina A, Sparti MG, Tamburini A and Micale G (2012) Development of a Membrane Distillation module for solar energy seawater desalination, Chem Eng Res Des 90 (12): 2101-2121.

[4] Francis L, Ghaffour N, Alsaadi AS and Amy GL (2013) Material gap membrane distillation: A new design for water vapour flux enhancement, Journ Membr Sci 448: 240-247.

[5] Francis L, Venugopal J, Prabhakaran MP, Thavasi V, Marsano E and Ramakrishna S (2010) Simultaneous electrospin-electrosprayed biocomposite nanofibrous scaffolds for bone tissue regeneration, Acta Biomaterialia, 6 (10): $4100-4109$.

[6] Yoon K, Kim K, Wang X, Fang D, Hsiao BS and Chu B (2006) High flux ultrafiltration membranes based on electrospun nanofibrous PAN scaffolds and chitosan coating, Polymer 47: 2434-2441.

[7] Francis L, Nair AS, Jose R, Ramakrishna S, Thavasi V and Marsano (2011) Fabrication and characterization of dye-sensitized solar cells from rutile nanofibers and nanorods, Energy, 36(1): 627-632.

[8] Marsano E, Francis L and Giunco F (2010) Polyamide 6 nanofibrous nonwovens via electrospinning, Journ Appl Polym Sci 117(3): 1754-1765.

[9] Lijo F, Marsano E, Vijila C, Barhate RS, Vijay VK, Ramakrishna S and Thavasi, V (2011) Electrospun Polyimide/Titanium Dioxide Composite Nanofibrous 
Membrane by Electrospinning and Electrospraying, Journ Nanosci Nanotech 11( 2): 1154-1159.

[10] Huang ZM, Zhang YZ, Kotaki M and Ramakrishna S (2003) A review on polymer nanofibers by electrospinning and their applications in nanocomposites, Compos Sci Technol 63 (15): 2223-2253.

[11] Francis L, Giunco F, Balakrishnan A and Marsano E (2010) Synthesis, characterization and mechanical properties of nylon-silver composite nanofibers prepared by electrospinning, Curr Appl Phys 10(4): 1005-1008.

[12] Miao J, Miyauchi M, Simmon TJ, Dordick JS and Linhardt RJ (2010) Electrospinning of Nanomaterials and Applications in Electronic Components and Devices, Journal of Nanoscience and Nanotechnology 10: 5507-5519.

[13] Francis L, Balakrishnan A, Sanosh KP and Marsano E (2010) Characterization and tensile strength of HPC-PEO composite fibers produced by electrospinning, Mater Lett 64 (16): 1806-1808.

[14] Kaur S, Rana D, Matsuura T, Sundarrajan S and Ramakrishna S (2012) Preparation and characterization of surface modified electrospun membranes for higher filtration flux, Journ Membr Sci, 390-391: 235-242.

[15] Maab H, Francis L, Alsaadi AS, Aubry C, Ghaffour N, Amy GL and Nunes SP (2012) Synthesis and fabrication of nanostructured hydrophobic polyazole membranes for low-energy water recovery, Journ Membr Sci 423-424: 11-19.

[16] Essalhi M, Khayet M, Cojocaru C, García-Payo MC and Arribas P (2013) Response Surface Modeling and Optimization of Electrospun Nanofiber Membranes, The Open Nanoscience Journal 7: 8-17. 
[17] Al-Obaidani S, Curcio E, Macedonio F, Profio G, Al-Hinai H and Drioli E (2008) Potential of membrane distillation in seawater desalination: Thermal efficiency, sensitivity study and cost estimation, Journ Membr Sci 323 (1): 85-98.

[18] Lalia BS, Burrieza EG, Arafat HA and Hashaikeh R (2013) Fabrication and characterization of polyvinylidenefluoride-co-hexafluoropropylene (PVDF-HFP) electrospun membranes for direct contact membrane distillation, Journ Membr Sci 428: 104-115.

[19] Francis L, Maab H, AlSaadi A, Nunes S, Ghaffour N and Amy GL (2013) Fabrication of electrospun nanofibrous membranes for membrane distillation application, Desalination and Water Treatment, 51 (7-9): 1337-1343.

[20] Adham S, Hussain A, Minier J, Dores MR and Janson A (2013) Application of Membrane Distillation for desalting brines from thermal desalination plant, Desalination 314: 101-108.

[21] Bonyadi S and Chung TS, Highly porous and macrovoid-free PVDF hollow fiber membranes for membrane distillation by a solvent-dope solution co-extrusion approach, Journ Membr Sci 331(1-2): 66-74. 
[22] Zhu H, Wang H, Wang F, Guo Y, Zhang H and Chen J (2013) Preparation and properties of PTFE hollow fiber membranes for desalination through vacuum membrane distillation, Journ Membr Sci 446: 145-153.

[23] Yang X, Wang R, Shi L, Fane A G, Debowski M (2011) Performance improvement of PVDF hollow fiber-based membrane distillation process, Journ Membr Sci 369 (1-2): 437-447.

[24] Gryta M (2012) Wettability of polypropylene capillary membranes during the membrane distillation process, Chemical Papers 66 (2): 92-98.

[25] Li JM, Xu ZK, Liu ZM, Yuan WF, Xiang H, Wang SY and Xu YY (2003) Microporous polypropylene and polyethylene hollow fiber membranes. Part 3. Experimental studies on membrane distillation for desalination, Desalination 155 (2): $153-156$.

[26] Khayet M, Mengual JI, Matsuura T (2005), Porous hydrophobic/hydrophilic composite membranes: application in desalination using direct contact membrane distillation, Journ Membr Sci 252 (1-2): 101-113.

[27] Bonyadi S and Chung TS (2007), Flux enhancement in membrane distillation by fabrication of dual layer hydrophilic-hydrophobic hollow fiber membranes Journ Membr Sci 306: 134-146.

[28] Wang P and Chung TS (2013) A New-Generation Asymmetric Multi-Bore Hollow Fiber Membrane for Sustainable Water Production via Vacuum Membrane Distillation, Envir Sci Technolo 47(12): 6272-6278.

[29] Maab H, Al Saadi A, Francis L, Livazovic S, Ghaffour N, Amy GL and Nunes SP (2013) Polyazole Hollow Fiber Membranes for Direct Contact Membrane Distillation, Ind Eng Chem Res 52 (31): 10425-10429. 